\title{
Physics Essay: Six Baseless and Irrational Problems in Modern Cosmology
}

\author{
Zifeng $\mathrm{Li}^{1}$ \\ ${ }^{1}$ Petroleum Engineering Department, Yanshan University, Hebei, China \\ Correspondence: Zifeng Li, Petroleum Engineering Department, Yanshan University, Hebei, China. E-mail: \\ zfli@ysu.edu.cn
}

Received: October 7, 2015 Accepted: October 21, 2015 Online Published: October 24, 2015

doi:10.5539/apr.v7n6p56 URL: http://dx.doi.org/10.5539/apr.v7n6p56

\begin{abstract}
Analyzes the Big Bang theory, recession of galaxies, Hubble's law, multi-dimensional space, curved space and black hole in modern cosmology and points out that these six theories are all baseless and irrational, contrary to classical science. Promotes the use of plain view of the universe - the materialist view of space-time-mass-energy to study the universe. The observations and understanding of the universe are very limited now. Cosmology should be realistic, not based on irrational models.
\end{abstract}

Keywords: universe, big bang, recession of galaxies, Hubble's law, curved space, black hole

Cosmology is the study of the universe as a whole, and then extends to the status of human beings in the universe. The study of the universe has a very long period of history, involving science, philosophy and religion.

When Einstein's special relativity and general relativity is introduced into the study of cosmology, the modern cosmology is formed, including the Big Bang theory, the recession of galaxies, Hubble's law, multi-dimensional (> 3D) space, curved space and black holes. If the detailed scrutiny of these doctrines is studied, none of them can be justified. It is necessary to regain the Newtonian time and space in the research of modern cosmology.

\section{Big Bang Theory and Its Defects}

\subsection{Big Bang Theory}

In 1927, the Belgian physicist, Catholic priest George Lemaitre, based on Einstein's general theory of relativity, first proposed the Big Bang theory on the origin of the universe ( $\mathrm{Su}, 2000)$. The theory is that: "The universe is formed by a Big Bang occurred about 13.7 billion years ago from the singular point; before the explosion, all matter and energy in the universe were gathered together and concentrated into a small volume, almost zero, the temperature was extremely high, with great density; then the big bang occurred, the material is scattering, the universe is expanding, the temperature is declining, and later have appeared all the galaxies, stars, planets and even life in the universe. " This doctrine is supported by the alleged recession of galaxies, and the Hubble law.

\subsection{Defects of Big Bang Theory}

Since the universe originates from the singularity, then where does the singular point come from? Is nebula really in the back? Is Hubble's law correct? These are questions that supporters of the Big Bang theory cannot answer. So far, in the mainstream physics community, no one dares to say that they understand the theory of relativity, but in fact the theory of relativity is wrong (Li, 2008; Ohanian, 2008; Schlafly, 2011; Yang \& Pan, 2007; Qi, 2006; Liu, 2011; Zhao, 2012). To this end, the Big Bang theory is unfounded.

\section{Star Redshift Theory and Its Defects}

\subsection{Star Redshift Theory}

American astronomer Hubble calls the shift of a celestial body's spectrum to longer wavelengths (red) end as the Doppler red shift (Yu, 2003; Liu, 2005). Astronomical observations show that all the stars show the "red shift". If interpreted as a Doppler effect, this would show that celestial bodies separated from each other, and that the universe is expanding, with the galaxy's recession velocity: $\mathrm{V}=$ redshift magnitude of the speed of light. 


\subsection{Defects of Star Redshift Theory}

People on the Earth can only measure starlight frequency while starlight reaches the Earth. To know whether it produces a red shift, you must know the starlight frequency when the starlight left the stars, as well as possible interaction with other substances in the long journey. The supporters of star redshift theory cannot give these parameters. To this end, the starlight redshift theory is unfounded.

\section{Hubble's Law and Its Defects}

\subsection{Hubble's Law}

Hubble in 1929 obtained the empirical laws - the redshift of light from distant galaxies is proportional to their distance, $V=H_{0} \times d, V$ is the recession velocity, $d$ is the galaxy distance, $H_{0}$ is the Hubble constant. This is called Hubble's law or the Hubble effect.

\subsection{Defects of Hubble's Law}

1) Since the starlight redshift theory is unfounded, the recession velocity in Hubble's law has no basis.

2) Distance measurement is a determination in accordance with the principles of optics and trigonometry. Your two eyes and the objects form a triangle, so you can determine the distance. One eye is unable to determine the object's distance. Putting a lot of telescopes on the Earth for measuring the distance between the planets within the solar system is effective. For the distant stars in the universe, the Earth-based telescopes are equivalent to one eye. If a lot of telescopes are placed on the Earth, in terms of the vastness of the universe, they are basically on the distant planet- Earth connection line, the measurement results cannot be trusted.

3) Since the recession velocity is not credible, the galaxy distances are not credible, but then the Hubble constant is not credible, i.e., the Hubble's law cannot be trusted.

4) The Hubble constant has been repeatedly revised. Hubble's projection of today's age of the universe is about 2 billion years. However, people already know the age of the Earth is 4.5 billion years. Earth was born before the universe, which of course is a ridiculous result and cannot be accepted. Modern astronomy studies have shown that the "Hubble constant" is continuously changing, the so-called constant changing minimally in a certain period of time. So, when d increases, $H_{0}$ will get smaller, that is, the Hubble constant is not constant. Using an ever-changing constant to measure the age of the universe is obviously unscientific.

\section{Multidimensional Space, Space Bending, Black Hole and Their Defects}

Multidimensional space, space bending, black hole are all from or related to general relativity.

\subsection{Multidimensional Space Theory and Its Defects}

\subsubsection{Multidimensional Space Theory}

Some scholars believe that the space is four-dimensional; some scholars believe that the space is 5-dimensional; ...

\subsubsection{Defects of Multidimensional Space Theory}

The supporters of the multi-dimensional space have confused the distinction between mathematical space and physical space. When people ask the supporters of the multi-dimensional space "Why people can only see three-dimensional space?" The answer is "people are too stupid".

\subsection{Space Bending Theory and Its Defects}

\subsubsection{Space Bending Theory}

Einstein's special theory of relativity, the non-Euclidean geometry and general relativity reveal the spatial and temporal characteristics changes of material forms and mass density. Space gets curved by the gravitational pull.

\subsubsection{Defects of Space Bending Theory}

Space has no material properties, so how can it be bent?

\subsection{Black Hole Theory and Its Defects}

\subsubsection{Black Hole Theory}

The black hole is a very strong gravitational object, and even light cannot escape. It absorbs material without the release of substances. 


\subsubsection{Defects of Black Hole Theory}

If there is a black hole, the entire universe will be sucked into the black hole.

\section{Cosmology Research Status}

Although most modern cosmology theories are baseness and rational, six theories in modern cosmology - the Big Bang theory, the recession of galaxies, Hubble's law, multi-dimensional (> 3D) space, curved space and black holes are unfounded and without reason, contrary to classical science.

The stellar radiation observed on the Earth, some fired at the Earth through approximate straight line, some have changed direction through the interplanetary gravitational field, and some are absorbed and re-emitted, as shown in Figure 1. The celestial space observed by telescope mounted on the Earth and Earth satellite is only a tiny fraction of the universe. Appropriate assumptions are available based on very limited observations. Excessive speculation is likely to be fallacious.

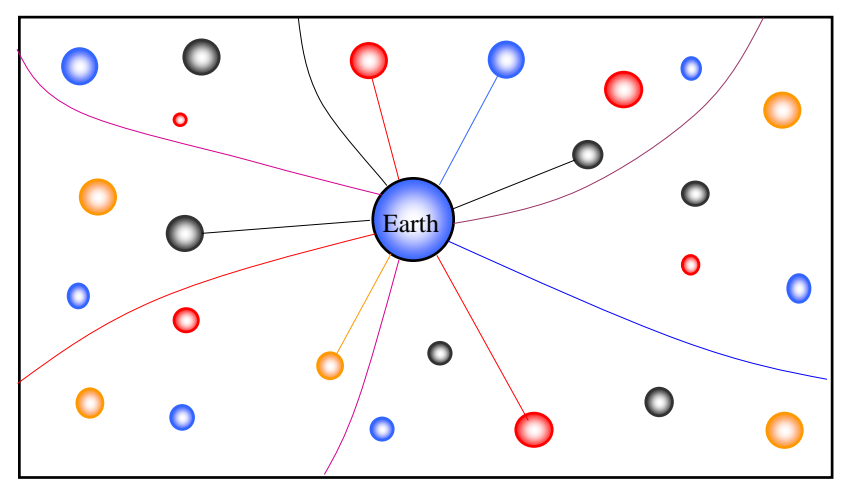

Figure 1. Starlight trajectories reaching the Earth

\section{Plain View of the Universe}

Since modern cosmology according to Einstein's general theory of relativity cannot be justified, it is necessary to regain the Newtonian time and space.

\subsection{Space-time-mass-energy Concept of Materialism}

1) Space. Space is one of the forms of materials' existence. It is infinite and boundless. Space is three dimensional and isotropic.

2) Time. Time is one of the forms of materials' existence. It is durance and sequential order of materials' process of motion. It is the objective existence not relying on people's consciousness and is eternal. Time is unidirectional, evenly flows without a beginning and end.

3) The relation between time and space. Time is time and space is space, they are all objectively existing. Time is not a function of space and space is not a function of time. They are fundamental elements of describing the material world, and can't be altered after their initial creation.

4) Mass. Mass is one of materials' essential attributes. It is the quantity of matter contained in an object. Object with zero mass does not exist. As long as it is a material, its mass must be greater than zero.

5) Energy. Energy is the state attribute of the material in motion. The energy of materials takes different forms. Under certain conditions, the materials' energy can transform mutually between the different forms, but the total energy is invariant.

6) The relation between energy and mass. Mass is mass and energy is energy. They are fundamental elements of describing matters and materials, and can't transform mutually into each other.

7) Mathematical space and physical space. In mathematics, each multi-dimensional variable may be seen as varying in the multi-dimensional space. In physics, there are the one-dimensional space (line), the two-dimensional space (surface) and the three-dimensional space (body) without any higher dimensional space. The multi-dimensional spaces of mathematics can't be transplanted directly to physics. If the dimension is smaller than or equal to three (not including time), then there is a correspondence between the mathematical space and the physical space. 
8) Source of atomic energy and the emission principle. Atomic energy comes from the energy internal to an atom. The emission of atomic energy is the transfer of photons together with their mass and energy. The mass and energy of the object decrease along with the release of energy from the object. The object, which receives energy, increases in its levels of energy and mass. The process of emitting atomic energy from an object is the same as a gun shooting a bullet, where the bullet is transferred together with mass and energy.

9) The universe is only one, there is no anti-universe.

10) The world is material, there is no antimatter; positively charged electrons are material, not antimatter.

11) Black holes (that absorb material without the release of substances) do not exist. White holes (that release material without absorbing material) do not exist.

12) Space is of no material property, and will not bend.

13) The passage of time is in constant rate and only forwards.

14) No Ether.

15) No action at a distance.

16) "God" Particle does not exist.

17) The laws of physics obey the law of causality.

\subsection{Four No Exact Answer Questions in Physics}

There are four no exact answer questions in physics. (1) The origin of the universe. The universe existed long before human, so the origin could not be verified. (2) The end of the universe. The universe will exist long after humans have vanished, so the end cannot be validated. (3) The scope of the universe. There can be no exact boundary. (4) How small material can be divided. From a philosophical perspective, it is infinitely divisible; from means of speaking, it is depends on the measurement techniques.

As for these problems, theoretical physicists and astronomers have to be realistic and only claim to know what can be known.

\section{Conclusion}

Six theories in modern cosmology - the Big Bang theory, the recession of galaxies, Hubble's law, multi-dimensional (> 3D) space, curved space and black holes are unfounded, there is no reason to them. Cosmology should be guided by the plain view of the universe. Cosmology should be realistic, and things only claimed to be known that already be known.

\section{Acknowledgment}

The paper is supported by the National Natural Science Foundation of China (NSFC) (Grant No. 51374183 and 51490653).

The author gratefully acknowledges Dr. Thomas Smid for polishing this paper's English.

\section{References}

Li, Z. F. (2008). Special relativity arising from a misunderstanding of experimental results on the constant speed of light. Physics Essays, 21(2), 96-102.

Liu, H. T. (2005). Study of absorption redshifts of quasars. Astronomical Research \& Technology, 2(03), 1-12.

Liu, Y. C. (2011). Special theory of relativity and its paradox (pp. 1-185). Beijing: Tsinghua University Press. (in Chinese)

Ohanian, H. C. (2008). Einstein's Mistakes: The Human Failings of Genius (pp. 1-329). New York: W. W. Norton \& Company, Inc.

Qi, J. (2006). New physics (pp. 95-162). Harbin: Northeast Forestry University Press. (in Chinese)

Schlafly, R. (2011). How Einstein Ruined Physics: Motion, Symmetry, and Revolution in Science (pp. 1-351). Dark Buzz.

Su, W. P. (2000). Evolution of the universe (pp. 187-199). Beijing: Peking University Press. (in Chinese)

Yang, G. L., \& Pan, M. N. (2007). Big comprehend science (pp. 211-250). Xi'An: Shanxi Science and Technology Press. (in Chinese)

Yu, Y. Q. (2003). Hot big bang cosmology (pp. 27-94). Beijing: Peking University Press. (in Chinese) 
Zhao, C. D. (2012). Foundations of physics research anthology (pp. 1-167). Chengdu: University of Electronic Science and Technology Publishing House. (in Chinese)

\section{Copyrights}

Copyright for this article is retained by the author(s), with first publication rights granted to the journal.

This is an open-access article distributed under the terms and conditions of the Creative Commons Attribution license (http://creativecommons.org/licenses/by/3.0/). 